\title{
High-level ab initio studies of unimolecular dissociation of the ground-state $\mathrm{N}_{3}$ radical
}

\author{
Peng Zhang and Keiji Morokumaa) \\ Cherry L. Emerson Center for Scientific Computation and Department of Chemistry, Emory University, \\ Atlanta, Georgia 30322
}

\author{
Alec M. Wodtke \\ Department of Chemistry and Biochemistry, University of California at Santa Barbara, Santa Barbara, \\ California 93106
}

(Received 14 July 2004; accepted 17 August 2004; published online 14 December 2004)

\begin{abstract}
A comprehensive study of the unimolecular dissociation of the $\mathrm{N}_{3}$ radical on the ground doublet and excited quartet potential energy surfaces has been carried out with multireference single and double excitation configuration interaction and second-order multireference perturbation methods. Two forms of the $\mathrm{N}_{3}$ radical have been located in the linear and cyclic region of the lowest doublet potential energy surface with an isomerization barrier of $62.2 \mathrm{kcal} / \mathrm{mol}$ above the linear $\mathrm{N}_{3}$. Three equivalent $C_{2 v}$ minima of cyclic $\mathrm{N}_{3}$ are connected by low barrier, meaning the molecule is free to undergo pseudorotation. The cyclic $\mathrm{N}_{3}$ is metastable with respect to ground state products, $\mathrm{N}\left({ }^{4} S\right)$ $+\mathrm{N}_{2}$, and dissociation must occur via intersystem crossing to a quartet potential energy surface. Minima on the seams of crossing between the doublet and quartet potential surfaces are found to lie substantially higher in energy than the cyclic $\mathrm{N}_{3}$ minima. This strongly suggests that cyclic $\mathrm{N}_{3}$ possesses a long collision-free lifetime even if formed with substantial internal excitation. (C) 2005 American Institute of Physics. [DOI: 10.1063/1.1804497]
\end{abstract}

\section{INTRODUCTION}

For nitrogen, unlike most elements, the energy of a N-N single bond is substantially less than one-third, and the energy of a $\mathrm{N}=\mathrm{N}$ double bond is substantially less than twothirds of a $\mathrm{N} \equiv \mathrm{N}$ triple bond. Consequently, all-nitrogen species that form rings at the expense of multiple bonds are subject to a strong chemical driving force toward dissociation to $\mathrm{N}_{2}$.

$$
N \stackrel{\Delta G \ll 0}{\longrightarrow} \quad N=N+N
$$

Not surprisingly, the ability of nitrogen to form cyclic structures has been difficult to observe directly or indirectly. Several experiments have led to the postulate of a cyclic polynitrogen species, but these have either remained unconfirmed or later been disproved. ${ }^{1-3}$ Recently, mass spectrometric detection of $\mathrm{N}_{5}^{-}$was attributed to a cyclic (aromatic) structure. ${ }^{4}$ Still, the possible existence of all-nitrogen cyclic allotropes has remained largely a topic for theory,,$^{2,5-10}$ and the question of how such molecules might be produced in the laboratory has remained open. ${ }^{11}$

Partly due to the fact that this class of molecules is so unstable, they have been vigorously sought as materials capable of storing large amounts of energy in minimal volumes, so-called "high energy and density materials." 12 Their natural tendency to decompose to $\mathrm{N}_{2}$ also makes them high on the list of environmentally friendly explosives and propellants.

\footnotetext{
a) Author to whom correspondence should be addressed. Electronic mail: morokuma@emory.edu
}

The simplest all-nitrogen ring is $\mathrm{N}_{3} \cdot{ }^{6-8}$ Recent highlevel calculations ${ }^{6}$ predict a $C_{2 v}$ molecule of $B_{1}$ symmetry and doublet spin-multiplicity with two long (1.455 $\AA$ ) and one short (1.218 ̊) NN bonds, molecule $\mathbf{1}$.

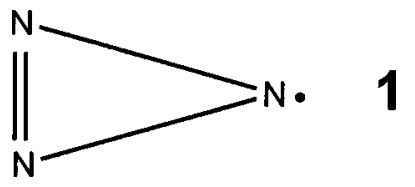

Isomerization to the well-known linear isomer (the azide radical) is predicted to be exoergic by $125 \mathrm{~kJ} / \mathrm{mol}$, spinallowed but limited by a $132 \mathrm{~kJ} / \mathrm{mol}$ barrier. ${ }^{6}$ Dissociation to ground state $\mathrm{N}\left({ }^{4} S_{3 / 2}\right)$ and $\mathrm{N}_{2}\left(\tilde{X}^{1} \Sigma_{g}^{+}\right)$is theoretically predicted to be exoergic by $125 \mathrm{~kJ} / \mathrm{mol}$, but is spin-forbidden. ${ }^{6}$ The lowest-energy spin-allowed dissociation channel forms $\mathrm{N}\left({ }^{2} D\right)$ and $\mathrm{N}_{2}\left(X^{1} \Sigma_{g}^{+}\right)$and is endoergic by $96 \mathrm{~kJ} / \mathrm{mol} .^{6}$ Theoretical calculations also suggest that the ${ }^{2} B_{1}$ cyclic- $\mathrm{N}_{3}$ may undergo barrierless exoergic recombination with $\mathrm{N}\left({ }^{2} D\right)$ atoms to form tetraazahedrane, ${ }^{1,2,5,6}$ molecule 2 . This makes cyclic- $\mathrm{N}_{3}$ one of the only suggested precursors to tetrahedral $-\mathrm{N}_{4} \cdot{ }^{10}$

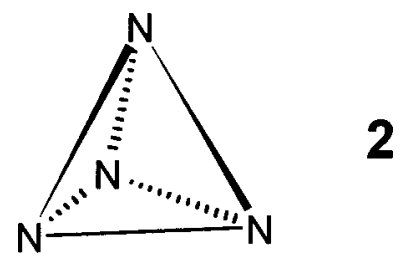

Tentative evidence for the photochemical production of cyclic $\mathrm{N}_{3}$ was recently reported by Hansen and Wodtke. ${ }^{13}$ In that work, velocity map ion images ${ }^{14}$ of $\mathrm{Cl}$ atoms recoiling of $\mathrm{CIN}_{3}$ after UV photon absorption exhibited a bimodal 
translational energy distribution, which was consistent with theoretical predictions of the heats of formation of cyclic and linear $\mathrm{N}_{3}$. While these experiments are indicative of the possible formation of cyclic- $\mathrm{N}_{3}$, the question of the stability of this molecule remains unanswered by experiment. Hansen and Wodtke ${ }^{13}$ were able to infer the energy content of the $\mathrm{N}_{3}$ formed in their experiments and argued that the majority was not formed with enough energy to dissociate the $\mathrm{N}\left({ }^{2} D\right)$ $+\mathrm{N}_{2}$.

These experiments make clear that the stability of cyclic $\mathrm{N}_{3}$ formed by photodissociation of $\mathrm{ClN}_{3}$ will ultimately be determined by the rate at which this molecule can decompose to $\mathrm{N}\left({ }^{4} S\right)+\mathrm{N}_{2}\left(\tilde{X}^{1} \Sigma_{g}^{+}\right)$, a spin-forbidden, albeit exoergic process involving curve-crossing dynamics.

While little if any experimental data is available concerning cylic- $\mathrm{N}_{3}$, copious experimental results on linear $\mathrm{N}_{3}$ have been reported. The electronic absorption spectrum was first measured by Thrush ${ }^{15}$ near $270 \mathrm{~nm}$, which was later assigned to an $\widetilde{A}^{2} \Sigma_{u} \leftarrow \tilde{X}^{2} \Pi_{g}$ transition by Douglas and Jones, ${ }^{16}$ and further confirmed by Beaman et al. using the laser-induced fluorescence technique. ${ }^{17}$ The structure of linear $\mathrm{N}_{3}$ was determined to be of $D_{\infty h}$ symmetry with a bond length of $1.18115 \AA$ by a Fourier transform IR (FTIR) study $^{18}$ and $1.181 \AA$ by analysis the rotational fine structure. ${ }^{16}$ For its vibrational frequency information, it has been reported by several groups using $\mathrm{CO}$ laser magnetic resonance ${ }^{19}$ and matrix FTIR ${ }^{20}$ techniques, respectively. The dissociation dynamics of excited $\mathrm{N}_{3}\left({ }^{2} \Sigma_{u}^{+}\right)$has also been investigated using fast radical beam photodissociation coupled with a coincidence wedge-and-strip-anode particle detector by Continetti et al. ${ }^{21,22}$ Both spin-allowed and spin-forbidden pathways were observed, however, the bend excitation in the ${ }^{2} \Sigma_{u}^{+}$state enhanced the spin-allowed process. Regarding the stability of linear $\mathrm{N}_{3}$, Pellerite et al. showed that $\mathrm{N}_{3}$ is roughly isoenergetic with $\mathrm{N}\left({ }^{4} S\right)+\mathrm{N}_{2}$ with $D_{0}\left(\mathrm{~N}_{2}-\mathrm{N}\right)=$ $-0.01 \pm 0.22 \mathrm{eV},{ }^{23}$ and a similar result, $D_{0}\left(\mathrm{~N}_{2}-\mathrm{N}\right)=$ $-0.05 \pm 0.10 \mathrm{eV}$, was obtained by Continetti et al. ${ }^{22}$

Quantum chemistry studies of both linear and cyclic- $\mathrm{N}_{3}$ have been performed at various levels of theory ranging from simple Hartree-Fock to highly correlated methods. To explain the observed absorption band in linear $\mathrm{N}_{3}$, configuration-interaction calculations have been carried out by both Petrongolo ${ }^{24}$ and Bittererova et al. ${ }^{6}$ At their best level of theory, Bittererova's MRCISD(Q)/AVTZ (multireference single and double excitation configuration interaction with augmented correlation consisted polarized valence triple zeta basis) energy of $4.55 \mathrm{eV}$ agrees well with the experimental value of $4.56 \mathrm{eV}$. The electronic excitation of cyclic $\mathrm{N}_{3}$ has also been examined by Bittererova et al. ${ }^{6}$ at MRCISD(Q)/AVTZ level.

The structure and vibrational frequencies of the $\mathrm{N}_{3}$ radical have been investigated by many theoretical groups for both the linear and cyclic forms. ${ }^{6,8,25}$ The ${ }^{2} B_{1}$ ring structure is found to be $29.9 \mathrm{kcal} / \mathrm{mol}$ above the linear isomer at UHF$\operatorname{CCSD}(\mathrm{T}) / \mathrm{VTZ}$ unrestricted coupled cluster level of theory. ${ }^{6}$ A low-lying quartet minimum $\left({ }^{4} B_{1}\right.$ open form structure) was found $40.6 \mathrm{kcal} / \mathrm{mol}$ above the linear ${ }^{2} \Pi_{g}$ at a small complete active space (CAS) (5e/5o)-CI/TZP level, ${ }^{8}$ and was determined to be the lowest quartet excited state. Other ring structures with different symmetries were shown to be high in energy (over $4 \mathrm{eV}$ ).

Regarding the molecule's stability, the dissociation energy to the spin-allowed product $\left[\mathrm{N}\left({ }^{2} D\right)+\mathrm{N}_{2}\right]$ was predicted to be $54.8 \mathrm{kcal} / \mathrm{mol}$ at MRAQCC (multireference average quadratic coupled cluster) level by Bittererova et al. ${ }^{6}$ and $51.9 \mathrm{kcal} / \mathrm{mol}$ by Petrongolo ${ }^{24}$ using a $[5 s 3 p 2 d]$ basis set, augmented by $(s p d)$ bond functions, and the MRD-CI procedure with nine reference configurations and energy extrapolation, and former value is closer to the experimental value of $54.9 \mathrm{kcal} / \mathrm{mol}^{22}$

Martin et al. ${ }^{26}$ using the G1 model, obtained a heat of formation for linear azide as follows: $\Delta H_{f, 0}^{0}\left(\right.$ linear $\left.\mathrm{N}_{3}\right)$ $=109.2 \pm 2 \mathrm{kcal} / \mathrm{mol}$. From this they obtained $D_{0}=+0.14$ $\pm 0.09 \mathrm{eV}$ (relative to $\left.\left[\mathrm{N}^{4} S\right)+\mathrm{N}_{2}\right]$ ), in reasonable agreement with the experiment. ${ }^{21-23}$ As for the cyclic $\mathrm{N}_{3}$, the dissociation transition state (TS) was determined to be of $C_{2 v}$ symmetry and $33 \mathrm{kcal} / \mathrm{mol}$ above the ${ }^{2} B_{1}$ ring minimum at $\operatorname{MRCISD}(\mathrm{Q}) / \mathrm{VTZ}$ level. ${ }^{6}$

Despite the large amount of theoretical work, there are still many unclear or misunderstood aspects of the electronic structure of the $\mathrm{N}_{3}$ radical, resulting from the spin- or symmetry-restricted nature of the previous calculations. In order to gain a more comprehensive understanding of the $\mathrm{N}_{3}$ molecule, including its ability to cyclize and its fundamental stability, we carried out a systematic study of the potentialenergy surfaces (PESs) of the ground doublet and quartet states using $a b$ initio molecular-orbital methods. The results of the study are presented in this paper, paying particular attentions to the spin-forbidden radiationless decay.

\section{METHODS OF CALCULATION}

Stationary points on the potential-energy surfaces have been optimized using both analytical and numerical gradient techniques at state-specific (ss-)CASSCF, CASPT2, and MRCISD(Q) levels with Dunning's augmented correlation consistent polarized valence triple and quadruple zeta basis sets (aug-cc-pVTZ and aug-cc-pVQZ). ${ }^{27}$ More specifically, at the complete active space self-consistent field $(\mathrm{CASSCF})^{28}$ level, the full valence active space, consisting of 15 electrons in 12 molecular orbitals and denoted as CAS(15e/12o), was used with fully uncontracted aug-cc-pVTZ (abbreviated as AVTZ). In the internally contracted second-order multireference perturbation-theory (CASPT2) calculations, ${ }^{29}$ the $1 s$ orbitals of the $\mathrm{N}$ atoms were kept doubly occupied in all configurations, and remaining 15 electrons were correlated, denoted as CASPT2(15e/12o) with the aug-cc-pVQZ (AVQZ) basis set. In CASPT2, we used Anderson's $g_{4}$ Fock operator, which makes CASPT2 calculations size extensive for cases where a molecule dissociates to high-spin openshell atoms, as zero-order Hamiltonian on top of a reference space at the CAS(15e/12o)/AVQZ level. At last, the internally contracted multireference configuration interaction calculations with single and double excitations (MRCISD) ${ }^{30}$ plus Davidson correction ${ }^{31}(\mathrm{Q})$ were performed with AVTZ using the reference configurations from the predetermined CAS(15e/12o)/AVTZ wave function with a CI coefficient threshold of 0.005 . Similar to CASPT2, only the $1 s$ orbitals 
TABLE I. Geometries and energetics of the linear $\mathrm{N}_{3}$ ground and low-lying excited states.

\begin{tabular}{|c|c|c|c|c|c|}
\hline & & $X^{2} \Pi_{g}$ & ${ }^{2} \Sigma_{u}^{+}$ & ${ }^{2} \Pi_{u}$ & $\tilde{a}^{4} \Pi_{u}$ \\
\hline \multirow{4}{*}{$\begin{array}{l}\text { Geometry } \\
(\AA)\end{array}$} & CASSCF & 1.1849 & 1.1849 & 1.2792 & 1.2790 \\
\hline & CASPT2 & 1.1843 & 1.1820 & 1.2718 & 1.2705 \\
\hline & $\operatorname{MRCISD}(\mathrm{Q})$ & 1.1854 & 1.1857 & 1.2693 & 1.2734 \\
\hline & Others & $1.18115,{ }^{\mathrm{d}} 1.184,{ }^{\mathrm{e}} 1.1831^{\mathrm{f}}$ & $1.184^{\mathrm{e}}$ & $\ldots$ & $\cdots$ \\
\hline \multirow{4}{*}{$\begin{array}{l}\text { Adiabatic energy }{ }^{\mathrm{a}} \\
(\mathrm{kcal} / \mathrm{mol})\end{array}$} & CASSCF & 0.00 & 112.28 & 111.17 & 102.28 \\
\hline & CASPT2 & 0.00 & 99.94 & 100.15 & 93.42 \\
\hline & MRCISD(Q) & 0.00 & 105.86 & 102.25 & 93.11 \\
\hline & Others & $\cdots$ & $105.04,^{\mathrm{g}} 108.01^{\mathrm{e}}$ & $104.23^{\mathrm{f}}$ & $\cdots$ \\
\hline \multirow{5}{*}{$\begin{array}{l}\text { Vertical excitation } \\
(\mathrm{kcal} / \mathrm{mol})\end{array}$} & CASPT2 & 0.00 & 98.48 & 113.97 & 109.00 \\
\hline & $\operatorname{MRCISD}(\mathrm{Q})$ & 0.00 & $105.87(0.0149)^{\mathrm{k}}$ & $117.38\left(9 \times 10^{-5}\right)^{\mathrm{k}}$ & $108.56(0.0)$ \\
\hline & Others & $\cdots$ & $\ldots$ & $115.76^{\mathrm{f}}$ & $\ldots$ \\
\hline & $\omega_{1}\left(\Pi_{u}\right)$ & $504.8,599.9(554.4)^{\mathrm{c}}$ & 629.2 & $443.3 i, 275.7 i$ & $278.7,1133.9 i$ \\
\hline & Others & $427.7,{ }^{\mathrm{h}} 457,,^{\mathrm{i}} 501,{ }^{\mathrm{f}}(528)^{\mathrm{e}}$ & $607^{e}$ & $\cdots$ & $\ldots$ \\
\hline \multirow{4}{*}{$\begin{array}{l}\text { Vibrational } \\
\text { frequency } \\
\left(\mathrm{cm}^{-1}\right)\end{array}$} & $\omega_{2}\left(\Sigma_{g}\right)$ & 1322.1 & 1322.0 & 1036.0 & 1068.4 \\
\hline & Others & $1287,{ }^{\mathrm{h}} 1320^{\mathrm{i}}$ & $1327^{\mathrm{e}}$ & $\ldots$ & $\ldots$ \\
\hline & $\omega_{3}\left(\Sigma_{u}\right)$ & 1649.2 & 2159.4 & 1876.5 & 1078.7 \\
\hline & Others & $1657.5^{\mathrm{h}} 1644.7^{\mathrm{j}}$ & $2155^{\mathrm{e}}$ & $\ldots$ & $\ldots$ \\
\hline
\end{tabular}

Zero-point vibrational energy (ZPE) was evaluated at CASSCF(15e/12o)/aug-cc-pVTZ level.

${ }^{b}$ Vibrational frequency was calculated at $\operatorname{CASSCF}(15 \mathrm{e} / 12 \mathrm{o}) / \mathrm{aug}-\mathrm{cc}-\mathrm{pVTZ}$ level.

${ }^{\mathrm{c}}$ The number in the parentheses is the harmonic vibrational frequency, which was evaluated as $\omega=\left\{0.5 \times\left[\left(\omega^{+}\right)^{2}+\left(\omega^{-}\right)^{2}\right]\right\}^{1 / 2}$.

${ }^{\mathrm{d}}$ Experimental values from Ref. 18.

'Theoretical values from Ref. 36: Geometry and frequency are at CASSCF level and selected MRCISD levels for energetic.

${ }^{\mathrm{f}}$ Theoretical values from Ref. 6: Geometry and frequency are at CASSCF/cc-pVTZ level, and selected MRCISD(Q)/aug-cc-pVTZ for energetic.

${ }^{\mathrm{g}}$ Experimental values from Refs. 17 and 21.

${ }^{\text {h}}$ Experimental values from Ref. 20.

${ }^{\mathrm{i}}$ Experimental values from Ref. 17.

${ }^{\mathrm{j}}$ Experimental values from Ref. 19.

${ }^{\mathrm{k}}$ The number in the parentheses is the oscillator strength evaluated at MRCISD(Q)/aug-cc-pVTZ level.

of $\mathrm{N}$ were kept doubly occupied in all configurations and remaining 15 electrons were correlated, denoted as $\operatorname{MRCISD}(\mathrm{Q})(15 \mathrm{e} / 12 \mathrm{o})$.

To characterize the nature of the equilibrium geometry and to determine the zero-point energy (ZPE), the Hessian matrix and vibrational frequencies were calculated by numerical differentiation of the energy gradient at the CAS(15e/12o)/AVTZ level on the CAS(15e/12o)/AVTZ optimized geometries, and this CASSCF ZPE will be used also for the ZPE correction at CASPT2(15e/12o)/AVQZ and $\operatorname{MRCISD}(\mathrm{Q})(15 \mathrm{e} / 12 \mathrm{o}) / \mathrm{AVTZ}$ levels. We also mention that the vertical excitation energies were calculated by CASPT2 and MRCISD(Q) methods using the two-state-averaged (with equal weight) (2sa-)CASSCF molecular orbitals, so that the transition moment and hence the oscillator strength can be calculated.

Minima on the seam of crossing (MSX) have also been optimized at the same CASSCF, CASPT2, and MRCISD(Q) levels using analytical and numerical techniques, respectively. In addition, 2sa-CASSCF method was also employed to optimize the MSXs in order to generate a wave fuction used in the spin-orbit interaction evaluations with full BreitPauli Hamiltonian approach ${ }^{32}$ at the two-state-averaged CASSCF optimized doublet-quartet crossing geometries.

MOLPRO 2002.6 ${ }^{39}$ was used for all the CASSCF, CASPT2, and MRCISD $(\mathrm{Q})$ calculations, except that two-stateaveraged CASSCF spin-orbit coupling calculations were performed by GAMESS-US 2000. ${ }^{33}$ Our own SEAM program in conjunction with HONDO 8.5 was employed for MSXs search. $^{34}$

\section{RESULTS AND DISCUSSION}

In this work, all energies will be expressed in $\mathrm{kcal} / \mathrm{mol}$ relative to the ground state linear $\mathrm{N}_{3}\left({ }^{2} \Pi_{g}\right)$ radical, unless otherwise specified. Two types of energies will appear in the discussion, one is ZPE corrected and the other in the parentheses is without ZPE correction. None of minima on the seam of crossing is corrected for ZPE. For the convenience of future discussion, the three $\mathrm{N}$ atoms will be labeled as $\mathrm{N}^{1}-\mathrm{N}^{2}-\mathrm{N}^{3}$. CASSCF will refer to calculations at CASSCF(15e/12o)/AVTZ level, CASPT2 stands for the calculation at CASPT2(15e/12o)/AVQZ level and MRCISD refers to the MRCISD(Q)(15e/12o)/AVTZ calculations. MRCISD energies will mainly be used for the discussion, unless otherwise specified.

\section{A. Linear stationary structures}

The electronic structure of ground-state linear $\mathrm{N}_{3}$ is ${ }^{2} \Pi_{g}$, with $\left(\sigma_{g}\right)^{2}\left(\sigma_{g}\right)^{2}\left(\sigma_{u}\right)^{2}\left(\sigma_{u}\right)^{2}\left(\pi_{u}\right)^{4}\left(\pi_{g}\right)^{3}\left(\pi_{u}^{*}\right)^{0}$ valence configuration, with the odd electron in the doubly degenerate nonbonding $\pi_{g}$ orbitals. The low-lying doublet excited states are $\widetilde{A}^{2} \Sigma_{u}^{+}$and $\widetilde{B}^{2} \Pi_{u}$, which originate from the $\pi_{g} \leftarrow \sigma_{u}$ and $\pi_{g} \leftarrow \pi_{u}$ transitions, respectively, and the lowest quartet state $\tilde{a}^{4} \Pi_{u}$ results from the $\pi_{u}^{*} \leftarrow \pi_{g}$ transition. The optimized structures, adiabatic energies, and harmonic vibrational frequencies of various excited states for linear structure at various theoretical levels along with experimental and previous theoretical results are shown in Table I. The vertical excitation energies at the geometries, respectively, optimized at different theoretical levels and the MRCISD oscillator 
strengths are also given in Table I. In general, our calculated results agree well with both experimental data as well as other theoretical values. For the ground state $\widetilde{X}^{2} \Pi_{g}$, the calculated bond length of $1.185 \AA$ at the MRCISD level, which is very close to those with CASSCF and CASPT2 levels, is $\sim 0.004 \AA$ longer than the experimental value of $1.181 \AA$ derived from Fourier transform spectroscopy. ${ }^{18}$ The computed harmonic stretching frequencies of $1322.1\left(\omega_{2}\right)$ and $1649.2\left(\omega_{3}\right) \mathrm{cm}^{-1}$ are very close to experimental fundamental frequencies measured with different techniques, such as $1287\left(\omega_{2}\right)$ and $1657.5\left(\omega_{3}\right)$ with matrix isolation, ${ }^{20} 1644.7$ for $\omega_{3}$ from laser magnetic-resonance infrared spectroscopy, and 1320 for $\omega_{2}$ from laser-induced florescence. ${ }^{17}$ However, for the average bending harmonic frequency $\left(554.4 \mathrm{~cm}^{-1}\right)$, which is evaluated as $\omega=\sqrt{1 / 2\left[\left(\omega^{+}\right)^{2}+\left(\omega^{-}\right)^{2}\right]}$, where $\omega^{+}$ and $\omega^{-}$correspond to nondegenerated harmonic frequencies caused by Renner-Teller interaction, ${ }^{35}$ is quite different from experimental fundamental value of 427.7 and $457 \mathrm{~cm}^{-1}$, most likely due to very anharmonic nature of this vibration.

For the $\widetilde{A}^{2} \Sigma_{u}^{+}$state, the calculated bond length is found to be the same as in the ground state at CASSCF level, and is only slightly different at CASPT2 and MRCISD levels, which is consistent with the result of rotational analysis of the spectra by Douglas and Jones. ${ }^{16}$ The reason for this similarity in bond length could be the result of the nature of electronic transition. Promoting an almost nonbonding $\sigma_{u}$ electron into a nonbonding $\pi_{g}$ orbital will not change the bonding character and therefore the bond length, which agrees with CASSCF and MRCISD results. However, CASPT2 results predict some bond distance change that may not be correct. The vibrational frequencies of bending modes increased upon excitation, consistent with previous theoretical calculation, ${ }^{36}$ presumably because the extra electron is placed in a $\pi$ orbital of the terminal $\mathrm{N}$ atoms causing repulsion upon bending. The calculated ZPE-corrected adiabatic energy, $105.9 \mathrm{kcal} / \mathrm{mol}$, at MRCISD level is only $0.9 \mathrm{kcal} /$ mol larger than the experimental value of $105.0 \mathrm{kcal} / \mathrm{mol}$, ${ }^{15}$ and is better than the previous selected MRCISD (without the $Q$ correction using a comparable basis set) value of 108.0 $\mathrm{kcal} / \mathrm{mol} .{ }^{36}$ CASPT2 result of $99.9 \mathrm{kcal} / \mathrm{mol}$ obviously underestimates this energy separation. Since the geometries of ground and excited states have very small difference, the vertical excitation energy, in principle, would be equal to adiabatic energy separation. The calculated MRCISD value using (2sa-)CASSCF wave function with the previously-used CASSCF equilibrium ZPE is $105.9 \mathrm{kcal} / \mathrm{mol}$, the same as the adiabatic excitation energy mentioned previously. The oscillator strength at MRCISD level is 0.0149, which is smaller than the previous CASSCF value of $0.0226 .^{6}$

There are few previous theoretical studies concerning the $\widetilde{B}^{2} \Pi_{u}$ state. The optimized bond length is $1.2693 \AA$ at MRCISD level, and CASSCF and CASPT2 calculations give the similar results with small difference. Frequency calculation at CASSCF level shows that both of bending modes are imaginary, which indicates $D$-type Renner-Teller splitting. ${ }^{35}$ These two components will relax and, finally, reach some bent minima on the PES, which is not the major concern in the current study, and will be discussed in a separate paper. At the linear stationary point, MRCISD as well as CASSCF calculations predict the lower energy for the $\widetilde{B}$ state, however, CASPT2 gives the reversed order. More reliable MRCISD result suggests that predissociation to $\widetilde{B}$ state could occur in the photodissociation. Vertical excitation energy is calculated to be 114.0 and $117.4 \mathrm{kcal} / \mathrm{mol}$ at CASPT2 and MRCISD levels, respectively, and these numbers agree well with previous theoretical value of $115.8 \mathrm{kcal} / \mathrm{mol}^{6}$ except, the present result has a little larger oscillator strength of 9.0 $\times 10^{-5}$.

The lowest linear quartet state, $\tilde{a}^{4} \Pi_{u}$, was first studied by Wasilewski. ${ }^{8}$ Our optimized bond length at all computational levels is $\sim 0.015 \AA$ longer than previous CASCI result of $1.259 \AA$. The calculated adiabatic energy separation is $93.1 \mathrm{kcal} / \mathrm{mol}$, which is $4.3 \mathrm{kcal} / \mathrm{mol}$ higher than previous CASCI energy. Vibrational calculation shows that there is an imaginary frequency $\left(1133.9 i \mathrm{~cm}^{-1}, b_{1}\right)$ in the bending mode, which represents the $C$-type Renner-Teller splitting, ${ }^{35}$ and will result in a ${ }^{4} B_{1}$ minimum (discussed later). Vertical excitation of $108.6 \mathrm{kcal} / \mathrm{mol}$ at MRCISD level indicates that $\tilde{a}^{4} \Pi_{u}$ lies inbetween $\widetilde{A}$ and $\widetilde{B}$ states but closer to $\widetilde{A}$. Combining adiabatic energy separation, one could expect the predissociation to the spin-forbidden product, which was observed in the photodissociation experiment by Continetti et al. $^{21}$

\section{B. Nonlinear stationary structures}

The optimized nonlinear stationary points on the ground doublet state $\left(D_{0}\right)$, quartet state $\left(Q_{1}\right)$, and MSXs between doublet and quartet states are shown in Figs. 1 and 2, respectively, and their energies are shown in Table II. The potential energy profiles are shown in Fig. 3. Frequency calculations indicate that not all of them are minima on the potential energy surfaces. The cyclic minimum, $D_{0_{-}}{ }^{2} B_{1}$, on the $D_{0}$ state is known from previous theoretical studies. ${ }^{8}$ Our optimized structures at CASSCF, CASPT2, and MRCISD levels are in excellent agreement with previous CASSCF, unrestricted second-order Møller-Plesset, Beck three parameter Lee-Yang-Parr, and $\operatorname{CCSD}(\mathrm{T})$ results, and energetically, it is lying $30.3(32.2) \mathrm{kcal} / \mathrm{mol}$ above the linear $\mathrm{N}_{3}$ global minimum, which is also in reasonable agreement with previous UHF-CCSD(T)/cc-pVTZ value of $29.9 \mathrm{kcal} / \mathrm{mol}^{6}$

Here, one may notice that the electronic structures of the cyclic ground-state structure $D_{0_{-}}{ }^{2} B_{1}$ and the linear groundstate structure $\tilde{X}^{2} \Pi_{g}$ are quite different. We will analyze the difference in detail by following orbitals and dominant configurations as structure is bent from linear

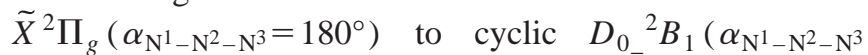
$=49.8^{\circ}$ ). As shown in Fig. 4, as $\alpha_{\mathrm{N}^{1}-\mathrm{N}^{2}-\mathrm{N}^{3}}$ decreases from $180^{\circ}$, the $\pi_{g}$ orbital split into two components, $4 b_{2}$ and $1 a_{2}$. Actually, the energy of $4 b_{2}$ climbs faster than that of $1 a_{2}$ and therefore the ground state is the ${ }^{2} B_{2}$ state $\left(1 b_{1}\right)^{2}\left(1 a_{2}\right)^{2}\left(4 b_{2}\right)^{1}$ with the ${ }^{2} A_{2}$ state $\left(1 b_{1}\right)^{2}\left(4 b_{2}\right)^{2}\left(1 a_{2}\right)^{1}$ slightly above. Around $\alpha_{\mathrm{N}^{1}-\mathrm{N}^{2}-\mathrm{N}^{3}}=110^{\circ}$, the vacant $6 a_{1}$ orbital that is used to be an in-plane component of $\pi_{u}^{*}$ antibonding orbital changes its character to $\mathrm{N}^{1}-\mathrm{N}^{2}$ and $\mathrm{N}^{2}-\mathrm{N}^{3}$ bonding orbital, lowers its energy very quickly, and becomes nearly degenerate with $4 b_{2}$ and $1 a_{2}$ orbitals. Here, the preferred configuration becomes $\left(1 b_{1}\right)^{2}\left(1 a_{2}\right)^{1}\left(4 b_{2}\right)^{1}\left(6 a_{1}\right)^{1}$, 


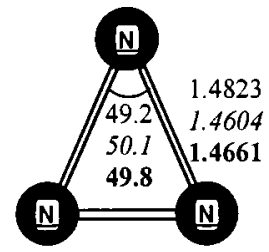

$\mathbf{D}_{0}{ }^{2} \mathbf{B}_{1}$ (Nimag $\left.=0\right)$

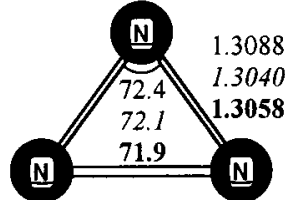

$\mathbf{D}_{0}{ }^{2} \mathbf{A}_{2} 1($ Nimag $=1)$

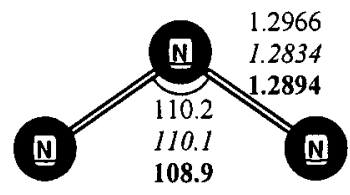

$\mathbf{D}_{0-}{ }^{2} \mathbf{A}_{2} 2$ (Nimag $\left.=2\right)$

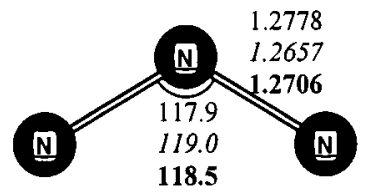

$\mathbf{Q}_{1}{ }^{4} \mathbf{B}_{1}(\mathrm{Nimag}=0)$

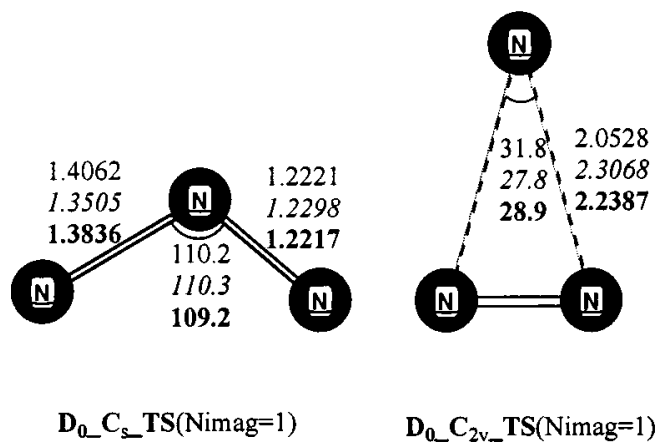

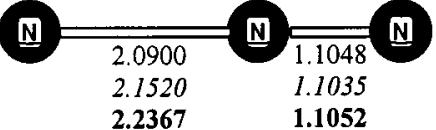

$\mathbf{D}_{0} \mathbf{C}_{\text {ovv_ }} \mathbf{T S}(\mathrm{Nimag}=1)$

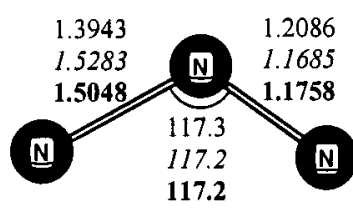

$\mathrm{Q}_{1} \mathrm{C}_{\mathrm{s}} \mathrm{TS}(\mathrm{Nimag}=1)$

FIG. 1. Stationary structures on the potential-energy surfaces. The plain, italic, and bold numbers refer to the optimized geometries at CASSCF, CASPT2, and MRCISD levels of theory, respectively.

resulting in the ${ }^{4} B_{1}$ ground state with the lowest doublet state ${ }^{2} B_{1}$ nearby. As the $\alpha_{\mathrm{N}^{1}-\mathrm{N}^{2}-\mathrm{N}^{3}}$ becomes even smaller, as shown in Fig. 4, the $6 a_{1}$ orbital lowers its energy further and at around $\alpha_{\mathrm{N}^{1}-\mathrm{N}^{2}-\mathrm{N}^{3}}=75^{\circ}$, the ground state becomes a new ${ }^{2} A_{2}$ state with the main configuration of $\left(1 b_{1}\right)^{2}\left(6 a_{1}\right)^{2}\left(1 a_{2}\right)^{1}$. Obviously, there is an interaction between the ${ }^{2} A_{2}$ configuration $\left(1 b_{1}\right)^{2}\left(4 b_{2}\right)^{2}\left(1 a_{2}\right)^{1}$ (dominant near linear structure) and the ${ }^{2} A_{2}\left(1 b_{1}\right)^{2}\left(6 a_{1}\right)^{2}\left(1 a_{2}\right)^{1}$ configuration (dominant in the $75^{\circ}$ structure), and the resultant avoided crossing should create a high barrier between the linear and cyclic structures of the $\mathrm{N}_{3}$ system.

Actually, calculations near this geometry have located a stationary structure with ${ }^{2} A_{2}$ symmetry, $D_{0_{-}}{ }^{2} A_{2_{-}} 2$ shown in Fig. 1. CASSCF frequency calculation indicates this is a second-order saddle point with two imaginary frequencies: 1310.1 $\left(a_{1}\right)$ and $1107.0\left(b_{2}\right) \mathrm{cm}^{-1}$. Moving along the $b_{2}$ coordinate, a $C_{s}$ stationary point, $D_{0_{-}} C_{S_{-}} \mathrm{TS}$, has been identified with only one imaginary frequency $\left(1018.3 \mathrm{~cm}^{-1}, a^{\prime}\right)$,

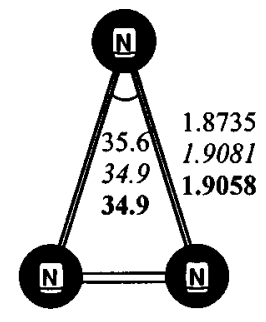

$\underline{39.67} \mathrm{~cm}^{-1}$

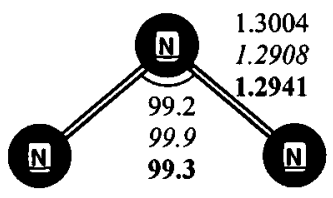

$\underline{20.84} \mathrm{~cm}^{-1}$

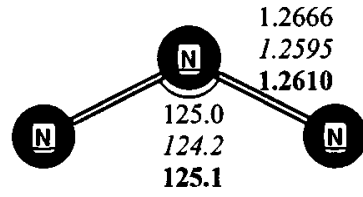

$\underline{26.84} \mathrm{~cm}^{-1}$

MSX_C $C_{2 v-}{ }^{2} A_{2} /{ }^{4} B_{1 \_} 2$

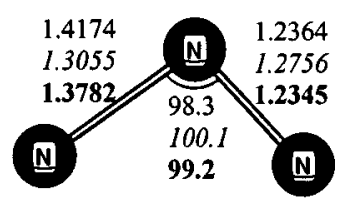

$\underline{20.90} \mathrm{~cm}^{-1}$

MSX_C ${ }_{\mathrm{s}-}{ }^{2} \mathrm{~A}^{\prime \prime} /{ }^{4} \mathrm{~A}^{\prime \prime}{ }_{-}$

$$
\text { MSX_C }{ }_{2 v_{-}}{ }^{2} B_{1} /{ }^{4} A_{2}
$$$$
\text { MSX_C } \mathrm{Cv}_{2}{ }^{2} \mathrm{~A}_{2} /{ }^{4} \mathbf{B}_{1} 1
$$

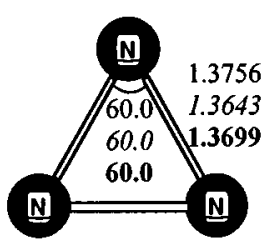

MSX_D ${ }_{3 \mathrm{~h} .}{ }^{2} \mathbf{B}_{1} /{ }^{2} \mathbf{A}_{2}$

FIG. 2. Optimized minima of seam of crossing (MSX) on the potential-energy surfaces. The plain, italic, and bold numbers refer to the optimized geometries at CASSCF, CASPT2, and MRCISD levels of theory, respectively. Numbers with underline are the normal of spin-orbit matrix. 
TABLE II. Energies (relative to the ground-state linear $\mathrm{N}_{3}{ }^{2} \Pi_{g}$ ) of various critical points on the doublet and quartet states of the $\mathrm{N}_{3}$ radical calculated with different $a b$ initio methods. ${ }^{\mathrm{a}}$

\begin{tabular}{|c|c|c|c|c|c|c|}
\hline & \multicolumn{5}{|c|}{ Relative energy $(\mathrm{kcal} / \mathrm{mol})$} & \multirow[b]{2}{*}{ Nimag; frequency $\left(\mathrm{cm}^{-1}\right)^{\mathrm{b}}$} \\
\hline & CASSCF & CASPT2 & $\operatorname{MRCISD}(\mathrm{Q})$ & Other work & $\mathrm{ZPE}^{\mathrm{b}}$ & \\
\hline${ }^{2} \Pi_{g}$ & -163.502185 & -163.919905 & -163.899074 & $\begin{array}{l}-163.88936^{\mathrm{c}} \\
-163.50097^{\mathrm{c}}\end{array}$ & 5.83 & 0 \\
\hline$D_{0_{-}}{ }^{2} B_{1}$ & 35.66 & 33.81 & 32.20 & $30.8,{ }^{\mathrm{d}} 29.9,{ }^{\mathrm{d}} 31.9^{\mathrm{d}}$ & 3.91 & 0 \\
\hline$D_{0_{-}}{ }^{2} A_{2} 1$ & 36.41 & 34.34 & 33.09 & $38.32^{\mathrm{e}}$ & 3.27 & $380.6\left(b_{2}\right)$ \\
\hline$D_{0_{-}}{ }^{2} A_{2}{ }^{2}$ & 71.03 & 67.21 & 66.07 & $\cdots$ & 1.71 & $1310.1\left(a_{1}\right), 1107.0\left(b_{2}\right)$ \\
\hline$D_{0_{-}} C_{S_{-}} \mathrm{TS}$ & 69.32 & 66.97 & 65.05 & $62.3,{ }^{\mathrm{f}} 65.1^{\mathrm{f}}$ & 3.16 & $1018.3\left(a^{\prime}\right)$ \\
\hline$D_{0_{-}} C_{2 v_{-}} \mathrm{TS}$ & 59.60 & 72.04 & 65.72 & $63.6^{\mathrm{g}}$ & 3.49 & $460.7\left(a_{1}\right)$ \\
\hline$D_{0_{-}} C_{\infty_{-}} \mathrm{TS}$ & 51.87 & 68.94 & 61.76 & $\cdots$ & 3.71 & $261.1\left(\Sigma_{u}\right)$ \\
\hline$Q_{1_{-}}{ }^{4} B_{1}$ & 49.36 & 46.99 & 46.18 & $56.5,{ }^{\mathrm{h}} 40.6^{\mathrm{h}}$ & 3.47 & 0 \\
\hline$Q_{1_{-}} C_{s_{-}} \mathrm{TS}$ & 49.54 & 51.27 & 48.69 & $\ldots$ & 3.00 & $390.4\left(a^{\prime}\right)$ \\
\hline $\operatorname{MSX} C_{2 v_{-}}{ }^{2} B_{1} /{ }^{4} A_{2}$ & 55.99 & 62.94 & 58.56 & $\cdots$ & $\cdots$ & $\ldots$ \\
\hline MSX_ $C_{2 v_{-}{ }^{2}} A_{2} /{ }^{4} B_{1} 1$ & 62.06 & 59.60 & 59.10 & $\cdots$ & $\cdots$ & $\cdots$ \\
\hline MSX_ $C_{2 v_{-}}{ }^{2} A_{2} /{ }^{4} B_{1}{ }^{2}$ & 50.73 & 47.72 & 47.39 & $\cdots$ & $\cdots$ & $\cdots$ \\
\hline MSX_ $C_{S_{-}}{ }^{2} A^{\prime \prime} /{ }^{4} A^{\prime \prime}{ }_{-}$ & 62.27 & 59.49 & 58.90 & $\cdots$ & $\cdots$ & $\cdots$ \\
\hline MSX_ $C_{S_{-}}{ }^{2} A^{\prime \prime} I^{4} A^{\prime \prime} \_2$ & 45.07 & 58.05 & 52.47 & $\cdots$ & $\cdots$ & $\cdots$ \\
\hline MSX_C $C_{S_{-}}{ }^{2} A^{\prime} /^{4} A^{\prime \prime}$ & 45.56 & 58.06 & 52.45 & $\cdots$ & $\cdots$ & $\ldots$ \\
\hline MSX $\_D_{3 h_{-}}{ }^{2} A_{2} /{ }^{2} B_{1}$ & 49.88 & 47.23 & 45.35 & $\ldots$ & $\cdots$ & $\cdots$ \\
\hline $\mathrm{N}\left({ }^{2} D\right)+\mathrm{N}_{2}$ & 44.74 & 64.42 & 58.98 & $56.6,{ }^{\mathrm{f}} 54.8^{\mathrm{f}}$ & 3.34 & 0 \\
\hline $\mathrm{N}\left({ }^{4} S\right)+\mathrm{N}_{2}$ & -18.42 & 10.12 & 2.26 & $3.2^{\mathrm{i}}$ & 3.34 & 0 \\
\hline
\end{tabular}

The energy of the ground-state linear $\mathrm{N}_{3}^{2} \Pi_{g}$ is expressed in a.u. For all others, relative energy is measured from ${ }^{2} \Pi_{g}$ without ZPE correction.

${ }^{b}$ Zero-point energies and vibrational frequencies are calculated at the CASSCF(15e/12o)/aug-cc-pVTZ level. Nimag refers to the number of imaginary frequency. When nimag $\geqslant 1$, it is omitted, and only imaginary frequencies and corresponding symmetries are given.

${ }^{c}$ Reference 6 at CAS $(15 e, 120) / c c-p V T Z$ and UHF-CCSD(T)/cc-pVTZ.

${ }^{d}$ Reference 6 at UHF-CCSD(T)/cc-pVTZ, MRCISD(Q)(0.025)/cc-pVTZ, and MRAQCC $(0.025) / c c-p V T Z$.

${ }^{\mathrm{e}}$ Reference 6 at CAS(11e,10o)/cc-pVTZ.

${ }^{\mathrm{f}}$ Reference 6 at MRCISD $(\mathrm{Q})(0.025) / \mathrm{cc}-\mathrm{pVTZ}$, and MRAQCC(0.025)/cc-pVTZ.

${ }^{\mathrm{g}}$ Reference 6 at MRCISD(Q)(0.025)/cc-pVTZ.

${ }^{\mathrm{h}}$ Reference 8 at UMP2/6-31G(d) and CASCI/TZP.

${ }^{\mathrm{i}}$ Reference 26 at the G1 model.

which is the real isomerization transition state connecting the linear and the cyclic $\mathrm{N}_{3}$, confirmed by intrinsic reaction coordinate (IRC) calculations performed at CASSCF level. At MRCISD level, $D_{0_{-}} C_{s_{-}}$TS lies 63.4 (65.1) and 32.1 (32.9) $\mathrm{kcal} / \mathrm{mol}$ above the $\tilde{X}^{2} \Pi_{g}$ and $D_{0}{ }^{2} B_{1}$, respectively, which is consistent with previous MRCISD single-point value of $31.5 \mathrm{kcal} / \mathrm{mol}^{6}{ }^{6}$ The CSAPT2 values of 64.3 (67.0) and 32.4 (33.1) $\mathrm{kcal} / \mathrm{mol}$ for the corresponding processes agree well with MRCISD results, but seem to overestimate the isomerization barrier. Compared to $D_{0_{-}}{ }^{2} A_{2} 2, D_{0_{-}} C_{s_{-}}$TS only 1.2 $\mathrm{kcal} / \mathrm{mol}$ lower in energy before the ZPE correction, but 0.2 $\mathrm{kcal} / \mathrm{mol}$ higher if the ZPE is included.

Starting from $D_{0}{ }^{2} A_{-2} 2$ again, another stationary point with the $A_{2}$ symmetry, $D_{0_{-}}{ }^{2} A_{2} 1$, is reached if one follows the $a_{1}$ coordinate. CASSCF Hessian calculations give one imaginary frequency $\left(380.6 i \mathrm{~cm}^{-1}\right)$ with $b_{2}$ symmetry, and IRC calculations at the same level indicate that it connects two equivalent $D_{0_{-}}{ }^{2} B_{1}$ structures. Here, we should emphasize that these $C_{2 v}$ structures, $D_{0_{-}}{ }^{2} B_{1}\left(\alpha_{\mathrm{N}^{1}-\mathrm{N}^{2}-\mathrm{N}^{3}}=49.8^{\circ}\right)$ and $D_{0_{-}}{ }^{2} A_{2} 1\left(\alpha_{\mathrm{N}^{1}-\mathrm{N}^{2}-\mathrm{N}^{3}}=71.9^{\circ}\right)$, have different $C_{2}$ axis and they are correlated by pseudorotation. One could imagine also that on the pseudorotation coordinate, there exist three equivalent $D_{0}{ }^{2} B_{1}$ minima on the PESs as well as three transition states, $D_{0}^{-}{ }^{2} A_{2} 1$, connecting them. The energy of $D_{0}{ }^{2} A_{2} 1$ is very close to that of $D_{0}{ }^{2} B_{1}$. Both CASSCF and MRCISD calculations give a $0.9 \mathrm{kcal} / \mathrm{mol}$ energy difference, which is reduced to $0.1 \mathrm{kcal} / \mathrm{mol}$ if ZPE is included, whereas the CASPT2 result of $-0.2(0.3) \mathrm{kcal} / \mathrm{mol}$ seems to underestimate the barrier. Overall, these results confirm the nearly free pseudorotation among three $C_{2 v}$ minima.

If the molecule further bends in the $a_{1}$ coordinate, starting from $D_{0_{-}}{ }^{2} A_{2}$, it will reach a conical intersection, MSX $\_D_{3 h}{ }^{2} A_{2} /{ }^{2} B_{1}$, in $D_{3 h}$ symmetry, where the $1 a_{2}$ and $2 b_{1}$ orbitals become degenerate $\left(\mathrm{e}^{\prime \prime}\right)$, as also shown in Fig. 4. One can see clearly that after this conical intersection, the $2 b_{1}$ orbital becomes lower in energy than the $1 a_{2}$ orbital, which results in changing the wave function from ${ }^{2} A_{2}\left(1 b_{1}\right)^{2}\left(6 a_{1}\right)^{2}\left(1 a_{2}\right)^{1}$ to ${ }^{2} B_{1}\left(1 b_{1}\right)^{2}\left(6 a_{1}\right)^{2}\left(2 b_{1}\right)^{1}$, and the latter one becomes the cyclic-N $\mathrm{N}_{3}$ minimum $\left(D_{0}{ }^{2} B_{1}\right)$ and the former corresponds to the pseudorotation transition state $\left(D_{0}{ }^{2} A_{2} 1\right)$. In fact, these two $C_{2 v}$ structures, $D_{0}{ }^{2} A_{2} 1$ and $D_{0}{ }^{2} B_{1}$, can be viewed as originating from the JahnTeller distortion of the degenerate ${ }^{2} E^{\prime \prime}$ states. The energy of this conical intersection is $13.2 \mathrm{kcal} / \mathrm{mol}$ above $D_{0}{ }^{2} B_{1}$ at the MRCISD level, and the calculated NN bond length is $1.370 \AA$ A. Now, combining all the information mentioned earlier the local PES picture around the cyclic $\mathrm{N}_{3}$ region would look like a Mexican hat.

The linear quartet $\mathrm{N}_{3}$ is a first-order saddle point on the PES. One component of the degenerate $\Pi_{u}$ state, ${ }^{4} B_{1}$, will go down in energy upon bending and, finally, reach a minimum, $Q_{1_{-}}{ }^{4} B_{1}$, which forms the lowest quartet PES. Frequency calculation confirms that there is no imaginary frequency, and energetically, it lies $43.8(46.2) \mathrm{kcal} / \mathrm{mol}$ above the linear ${ }^{2} \Pi_{g} \mathrm{~N}_{3}$. In the cyclic region, we have also found 


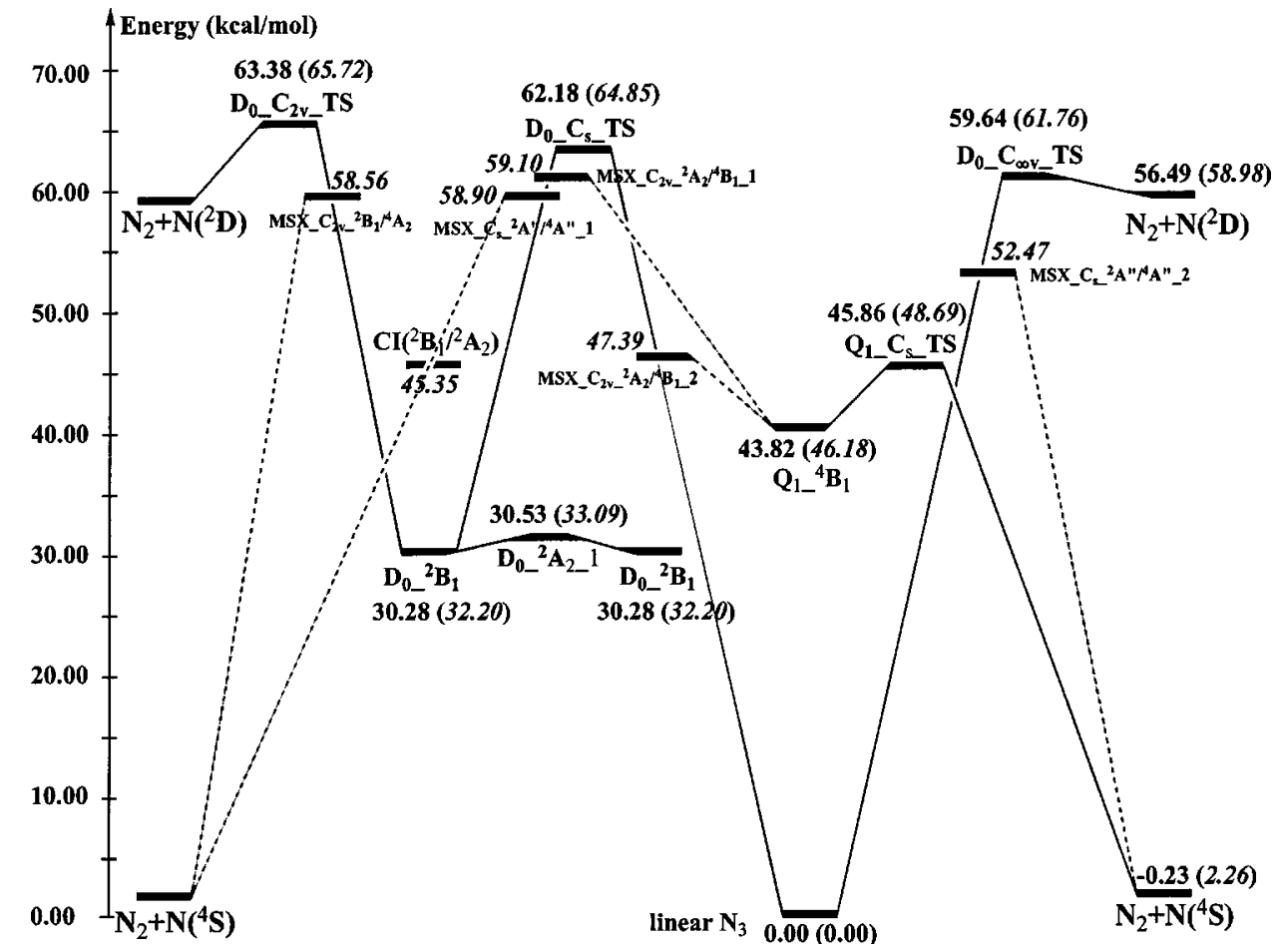

FIG. 3. MRCISD potential-energy profiles of the $\mathrm{N}_{3}$ radical. The plain numbers are ZPE corrected and italic numbers are not ZPE corrected. several quartet equilibrium structures; however, they are all high-energy species and correspond to structures on higher excited states. Simple PES scan indicates that the potential is purely repulsive in the cyclic region for the lowest quartet state.

\section{Adiabatic dissociation on the doublet and quartet potential-energy surfaces}

Before we start to discuss the unimolecular decomposition process of the $\mathrm{N}_{3}$ species, let us first look at the dissociation energy of both linear and cyclic $\mathrm{N}_{3}$. The dissociation energies calculated at all computational levels using, respectively, optimized $\mathrm{N}_{3}$ and $\mathrm{N}_{2}$ structures are listed in Table II. Previously, two experiments have independently confirmed that linear $\mathrm{N}_{3},{ }^{2} \Pi_{g}$, is slightly endoergic relative to $\mathrm{N}\left({ }^{4} S\right)$ $+\mathrm{N}_{2}$ at $0 \mathrm{~K}$, with the dissociation energies of $D_{0}\left(\mathrm{~N}_{2}-\mathrm{N}\right)$

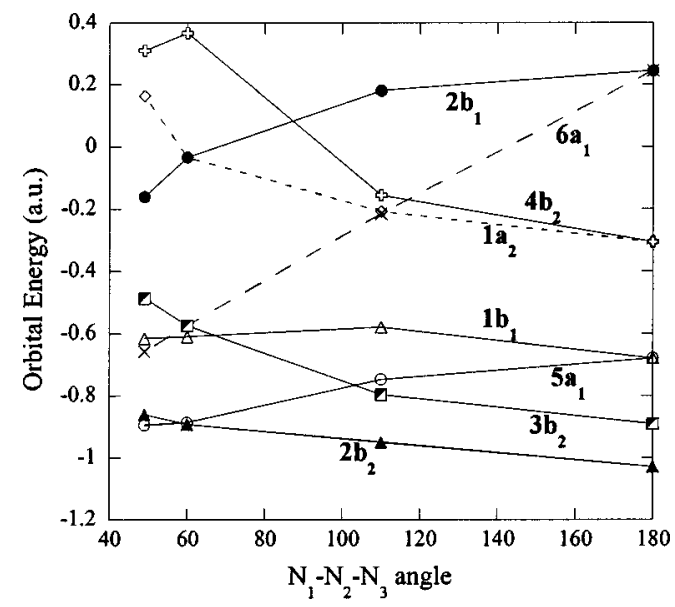

FIG. 4. CASSCF natural orbital energy changes with respect to the $\mathrm{N}^{1}-\mathrm{N}^{2}-\mathrm{N}^{3}$ angle.
$=-1.15 \pm 2.31^{22}$ and $D_{0}\left(\mathrm{~N}_{2}-\mathrm{N}\right)=-0.23 \pm 5.07^{23} \mathrm{kcal} / \mathrm{mol}$. Our calculated MRCISD result of -0.23 (2.26) $\mathrm{kcal} / \mathrm{mol}$ is in reasonable agreement with experimental findings. Also, the energy separation between the $\mathrm{N}\left({ }^{4} S\right)$ and $\mathrm{N}\left({ }^{2} D\right)$ is calculated to be $56.7 \mathrm{kcal} / \mathrm{mol}$, which is $1.7 \mathrm{kcal} / \mathrm{mol}$ higher than the experimental value of $55.0 \mathrm{kcal} / \mathrm{mol},{ }^{37}$ validating the methodology used in the study. However, CASPT2 and CAS results differ from experimental values by about $10-20 \mathrm{kcal} /$ mol although their $\mathrm{N}\left({ }^{4} S\right)$ and $\mathrm{N}\left({ }^{2} D\right)$ energy separations are quite close to experimental result. Therefore, MRCISD energies will mainly be used in the following discussions.

Adiabatically, the ${ }^{2} B_{1}$ state of cyclic $\mathrm{N}_{3}$ correlates directly to the $\mathrm{N}\left({ }^{2} D\right)+\mathrm{N}_{2}$ product, and a transition state, $D_{0} C_{2 v}$ TS, has been located with $C_{2 v}$ symmetry. Frequency calculation at the CAS level indicates that there is only one imaginary frequency $\left(461 i \mathrm{~cm}^{-1}, a_{1}\right)$ corresponding to the symmetric stretch motion, and IRC calculation confirms that this TS connects $D_{0_{-}}{ }^{2} B_{1}$ and $\mathrm{N}\left({ }^{2} D\right)+\mathrm{N}_{2}$ with the apical $\mathrm{N}$ atom dissociating. One can see that the CASSCF results agree well with the previous CASSCF studies. ${ }^{6}$ However, when applying the dynamically correlated methods, CASPT2 and MRCISD, the dissociation TS has two longer $\mathrm{N}-\mathrm{N}\left(\mathrm{N}^{1}-\mathrm{N}^{2}\right.$ and $\left.\mathrm{N}^{2}-\mathrm{N}^{3}\right)$ bonds, indicating the dynamic correlation makes the TS more productlike. Energetically, $D_{0} C_{2 v}$ TS is of $33.1(33.5) \mathrm{kcal} / \mathrm{mol}$ above the $D_{0_{-}}{ }^{2} B_{1}$, which corresponds to a recombination barrier of 6.2 (6.1) $\mathrm{kcal} / \mathrm{mol}$ at the MRCISD level, and this result is slightly lower than the previous $7 \mathrm{kcal} / \mathrm{mol}$ activation energy. ${ }^{6}$ Also, one can see that CASPT2 gives a $1.3 \mathrm{kcal} / \mathrm{mol}$ recombination barrier but a more reasonable dissociation barrier of $36.3 \mathrm{kcal} / \mathrm{mol}$. This relatively large difference in recombination barrier could be due to the error in correctly describing the dissociation limit using the CASPT2 method.

On the linear side, there is no previous work concerning 
the dissociation TS. The $\mathrm{N}_{3}{ }^{2} \Pi_{g}$ also correlates to the $\mathrm{N}\left({ }^{2} D\right)+\mathrm{N}_{2}$ product directly, and a transition state, $D_{0_{-}} C_{\infty v_{-}}$TS of $C_{\infty v}$ symmetry in Fig. 1 has been identified at all levels of theory. The major feature of this TS is the substantially elongated $\mathrm{N}-\mathrm{N}$ bond of $2.236 \AA$, and the only imaginary frequency $\left(1213 i \mathrm{~cm}^{-1}\right)$ at the CAS level corresponds to the asymmetric $\mathrm{N}-\mathrm{N}$ stretch. IRC calculation verified the connectivity of this TS. Energetically, $D_{0_{-}} C_{\infty v_{-}}$TS is $59.6(61.8) \mathrm{kcal} / \mathrm{mol}$ relative to the linear ground state, and $3.2(2.8) \mathrm{kcal} / \mathrm{mol}$ above the dissociation product at the MRCISD level. CASPT2 again gives a different result; the TS is $1.74 \mathrm{kcal} / \mathrm{mol}$ lower than the dissociation limit.

In a previous calculation, ${ }^{6}$ a new synthetic route to tetraazatetrahedrane $\left(\mathrm{N}_{4}\right)$ was proposed based on the reaction of cyclic- $\mathrm{N}_{3}$ with $\mathrm{N}\left({ }^{2} D\right)$. One might inquire if such a strategy could be applied to produce cyclic $\mathrm{N}_{3}$ through the reaction of $\mathrm{N}_{2}\left(\tilde{X}^{1} \Sigma_{g}^{+}\right)$with $\mathrm{N}\left({ }^{2} D\right)$. The current calculation indicates this might be an unlikely approach in the lowcollision energy condition. Rather, the linear isomer would more likely be the major product if $\mathrm{N}_{2}\left(\tilde{X}^{1} \Sigma_{g}^{+}\right)$reacted with $\mathrm{N}\left({ }^{2} D\right)$, because the MRCISD recombination barriers are 6.2 and $3.2 \mathrm{kcal} / \mathrm{mol}$ for the cyclic and linear side, respectively. However, at higher collision conditions, the small energy difference may be overcome and the cyclic $\mathrm{N}_{3}$ may be also formed.

The dissociation channel on the $Q_{1}$ state is also studied for the first time. The optimized TS, $Q_{1_{-}} \mathrm{TS}$, leading to $\mathrm{N}\left({ }^{4} S\right)+\mathrm{N}_{2}$ product is shown in Fig. 1. The TS is of $C_{s}$ symmetry, as the $\mathrm{N}\left({ }^{4} S\right)+\mathrm{N}_{2}$ product correlates to an ${ }^{4} A_{2}$ state in the $C_{2 v}$ symmetry. Compared with the $Q_{1}$ minimum, $Q_{1_{-}}{ }^{4} B_{1}, Q_{1_{-}}$TS is closer to the reactant side, with one of the $\mathrm{N}-\mathrm{N}$ bonds stretched from 1.271 to $1.505 \AA$ and the other $\mathrm{NN}$ bond and $\mathrm{N}^{1} \mathrm{~N}^{2} \mathrm{~N}^{3}$ bond angle changed very little. The only imaginary frequency $\left(459 i \mathrm{~cm}^{-1}, a^{\prime}\right)$ corresponds to the $\mathrm{N}-\mathrm{N}$ stretching mode. At the MRCISD level, the dissociation barrier is $2.0(2.5) \mathrm{kcal} / \mathrm{mol}$, suggesting an easy quartet dissociation and a short lifetime.

\section{Spin-orbit interaction-induced nonadiabatic dissociation}

So far, we have discussed the adiabatic dissociation within the lowest doublet and the lowest quartet surfaces. Since the energy of high spin (quartet) product is far below the doublet product, $56.4(56.7) \mathrm{kcal} / \mathrm{mol}$ at MRCISD level, one can expect that the intersystem crossing will occur during the unimolecular dissociation. Basically, three regions could contribute to the spin-forbidden process. The first two are in the vicinity of $\mathrm{N}_{3}$ dissociation transition states and the other one is around the linear to cyclic $\mathrm{N}_{3}$ isomerization transition state region, where the quartet $\mathrm{N}_{3}$ minimum, $Q_{1_{-}}{ }^{4} B_{1}$, is also located nearby.

The seam of crossing between two electronic states with different spin multiplicity occurs in up to $3 n-7$ dimensions, where $n$ is the number of atoms in the system. The minimum on the crossing seam hypersurface (MSX) is a critical point that gives the minimum energy required to make transitions between two states, and the efficiency of transition is determined by the magnitude of spin-orbit interaction, energy gradients, as well as the relative energies. A perturbation treatment suggests that the transition is dominant near the crossing, i.e., when two states have the same energy.

\section{ISC in the vicinity of cyclic $N_{3}$ dissociation TS}

As mentioned in the previous section, the $\mathrm{N}\left({ }^{4} S\right)+\mathrm{N}_{2}$ product correlates to the ${ }^{4} A_{2}$ or ${ }^{4} A$ " state in $C_{2 v}$ or $C_{s}$ symmetry, respectively. In the vicinity of cyclic $\mathrm{N}_{3}$ dissociation TS, $D_{0_{-}} C_{2 v_{-}} \mathrm{TS}$, a minimum on the crossing seam between doublet $\left({ }^{2} B_{1}\right)$ and quartet $\left({ }^{4} A_{2}\right)$ states, MSX_$C_{2 v_{-}}{ }^{2} B_{1} /{ }^{4} A_{2}$, of $C_{2 v}$ symmetry has been determined at all levels of theory and shown in Fig. 2. Compared to the doublet dissociation TS, $D_{0_{-}} C_{2 v_{-}} \mathrm{TS}$, the $\mathrm{N}^{1}-\mathrm{N}^{2}$ (or $\mathrm{N}^{2}-\mathrm{N}^{3}$ ) bond length in this MSX is about $0.33 \AA$ shorter and the $\mathrm{N}^{1}-\mathrm{N}^{2}-\mathrm{N}^{3}$ angle is $6.0^{\circ}$ larger, suggesting that crossing occurs on the dissociation coordinate and before the dissociation transition state. The energy of MSX_ $C_{2 v_{-}}{ }^{2} B_{1} /{ }^{4} A_{2}$ is $26.4 \mathrm{kcal} / \mathrm{mol}$ relative to the cyclic $\mathrm{N}_{3}$ minimum and $7.2 \mathrm{kcal} / \mathrm{mol}$ below the $C_{2 v}$ dissociation TS at MRCISD level without ZPE correction.

The norm of two state-averaged CASSCF spin-orbit coupling matrix elements,

$$
h^{s o}=\left\langle D_{0}\left({ }^{2} B_{1}\right)\left|H^{\mathrm{SO}}\right| Q_{1}\left({ }^{4} A_{2}\right)\right\rangle,
$$

at this MSX geometry is calculated to be $39.7 \mathrm{~cm}^{-1}$, implying a very strong doublet-quartet interaction. At the MSX geometry, the singly occupied orbital in the doublet wave function is largely an out-of-plane $\mathrm{N}^{2}\left(p_{x}\right)$ orbital oriented perpendicular to the molecular plane ( $y z$ plane), and the three singly occupied orbitals in the quartet wave function are largely the out-of-plane $\mathrm{N}^{2}\left(p_{x}\right)$ orbital and the $\mathrm{N}-\mathrm{N}$ inplane $\sigma\left(b_{2}\right)$ and $\sigma^{*}\left(a_{1}\right)$ orbitals, therefore, only integrals with $\Delta M_{\mathrm{S}}= \pm 1$ will survive.

It is also useful to consider the magnitude of spin-orbit coupling in the context of the propensity for the mechanistic bifurcation in the $\mathrm{N}_{3}$ unimolecular dissociation. This can be achieved using a Laudau-Zener ${ }^{38}$ approach, in which the ${ }^{2} B_{1}$ and ${ }^{4} A_{2}$ states are viewed as diabatic states coupled by spinorbit interaction. In such a model, the probability $P$ of a nonoadiabatic transition, i.e., the probability of remaining on a same diabatic PES, is given by

$$
P=\exp [-(\pi / 4) \xi],
$$

where the generalized Massey parameter ${ }^{38} \xi$ is given by

$$
\xi=8\left|h^{s o}\right|^{2} / \sum_{i} \frac{\partial}{\partial R_{i}}(\Delta E) v_{i}
$$

where $\left|h^{s o}\right|$ is the norm of spin-orbit matrix, $v$ is an appropriate nuclear velocity vector, and $i$ runs over $3 \mathrm{~N}-6$ internal coordinates. Pure adiabatic (spin-forbidden) motion corresponds to $\xi=\infty$ and $P=0$, while the diabatic (spin-allowed) limit corresponds to $\xi=0$ and $P=1$. Now, in the current system, the MRCISD calculations show that the gradient of $\Delta E$ is determined by the repulsive quartet potential and is by far largest for motion along the direction of $\mathrm{N}^{1}-\mathrm{N}^{2}-\mathrm{N}^{3}$ bending. Thus, the motion along this coordinates will, from the previous equation, tend to be more diabatic, spin conserving. From the calculations, the gradient difference for that coordinate was found to be 0.379 a.u. at the MRCISD level. Now, let's assume 1000 and $100 \mathrm{~cm}^{-1}$ kinetic energy along this 
coordinate, respectively; then, the probabilities of the spinconserving path are $P=0.999$ and $P=0.996$, respectively. Thus, only very near the threshold, for which $v_{i} \rightarrow 0$, will the probability of adiabatic, spin-forbidden behavior be appreciable at this MSX. Based on the mentioned information, we can estimate the lifetime of this species using the first-order reaction model. Symmetric stretch vibrational mode of 1629 $\mathrm{cm}^{-1}$ at CASSCF level is responsible for reaching this MSX, which suggests that this MSX is reached every vibrational period of $21 \mathrm{fs}$. Dividing the spin-forbidden transition probability $1-P$ by the vibrational period, one obtains the estimated spin-forbidden reaction rates $(k)$ of $\sim 5 \times 10^{10} \mathrm{~s}^{-1}$ and $\sim 2 \times 10^{11} \mathrm{~s}^{-1}$ for 1000 and $100 \mathrm{~cm}^{-1}$ kinetic energy, respectively. Correspondingly, lifetimes $(\tau=1 / k)$ are $\sim 20$ and $\sim 5$ ps for each kinetic energy. This result indicates that a substantial spin-forbidden dissociation can actually take place although the probability for a single transition is small. Note, however, that this is a very crude estimation; in particular, if the total internal energy of the cyclic species is not large enough, the system will not reach this MSX and will be long lived.

\section{ISC in the vicinity of isomerization TS}

In this region, there are two possibilities that quartet and doublet states could cross. One is before the isomerization TS and the other is after the isomerization TS. Here, we assume the isomerization process occurring from cyclic to linear $\mathrm{N}_{3}$; thus, the region closer to the cyclic minimum will be defined as before TS.

First, before the isomerization TS, two MSXs have been located with all methods used in this study. The one, MSX_ $C_{2 v}{ }^{2} A_{2} /{ }^{4} B_{1} 1$, of $C_{2 v}$ symmetry and shown in Fig. 2 , originates from the further bending of quartet minimum, $Q_{1_{-}}{ }^{4} B_{1}$, whose energy increases upon bending. Comparing geometries between $Q_{1_{-}}{ }^{4} B_{1}$ and MSX_C $C_{2 v_{-}}{ }^{2} A_{2} /{ }^{4} B_{1_{-}} 1$, one can see a large change in the $\mathrm{N}^{1}-\mathrm{N}^{2}-\mathrm{N}^{3}$ bond angle, compressed from $118.5^{\circ}$ to $99.3^{\circ}$, and a small shortening of 0.025 $\AA$ in $\mathrm{N}^{2}-\mathrm{N}^{3(1)}$ bond length. Also, compared to the isomerization TS, $D_{0_{-}} C_{S_{-}} \mathrm{TS}$, the $\mathrm{N}^{1}-\mathrm{N}^{2}-\mathrm{N}^{3}$ bond angle in MSX_ $C_{2 v_{-}}{ }^{2} A_{2} /{ }^{4} B_{1}{ }_{-} 1$ is about $10^{\circ}$ smaller, indicating that this MSX is located before the TS. At the MRCISD level, MSX_ $C_{2 v_{-}}{ }^{2} A_{2} / I^{4} B_{1} 1$ is about $6.0 \mathrm{kcal} / \mathrm{mol}$ (uncorrected for ZPE) lower in energy than $D_{0_{-}} C_{s_{-}} \mathrm{TS}$, which means 26.9 $\mathrm{kcal} / \mathrm{mol}$ (uncorrected for ZPE) above the cyclic $\mathrm{N}_{3}$ minimum, $D_{0_{-}}{ }^{2} B_{1}$. Calculations of these energy differences at the CASPT2 level of the theory yield 7.4 and $25.8 \mathrm{kcal} / \mathrm{mol}$.

CASSCF spin-orbit calculation at the state-averaged CASSCF-optimized MSX geometry gives $20.8 \mathrm{~cm}^{-1}$ for the norm of the coupling matrix, which also results from components with $\Delta M_{s}= \pm 1$. Analysis of the wave function shows that the contributions are from interactions between a nonbonding out-of-plane ( $x y$ plane) $\pi^{*}$ orbital and the two in-plane orbitals ( $\sigma^{*}$ and nonbonding $\pi^{*}$ ). The MRCISD gradient difference value of 0.248 a.u. indicates that the bending motion is the largest contribution to the spin-orbit transition. Applying the same Laudau-Zener model as before and assuming 1000 and $100 \mathrm{~cm}^{-1}$ kinetic energy again, one obtain probabilities of the spin-conserving path are $P$ $=0.999$ and $P=0.998$, respectively. Obviously, the contribu- tion will be still very small even when the kinetic energy approaches zero. Compared to MSX_C $C_{2 v_{-}}{ }^{2} B_{1} /{ }^{4} A_{2}$, MSX_ $C_{2 v_{-}}{ }^{2} A_{2} /{ }^{4} B_{1} 1$ is almost energetically equivalent to MSX_ $C_{2 v_{-}}{ }^{2} B_{1} / I^{4} A_{2}$, however, the one-dimensional LaudauZener model indicates that MSX_ $C_{2 v_{-}}{ }^{2} B_{1} /{ }^{4} A_{2}$ may contribute much more to dissociation. Applying the same procedure as mentioned earlier we obtain $\tau \approx 40$ and $20 \mathrm{ps}$ for the two kinetic energies, respectively, where the bending mode ( 826 $\mathrm{cm}^{-1}$ ) is responsible for reaching this MSX.

In addition to MSX_C $C_{2 v_{-}}{ }^{2} A_{2} /{ }^{4} B_{1} 1$, there is another crossing occurring before the isomerization TS. The optimized minimum on the seam of crossing hypersurface, MSX_ $C_{S_{-}}{ }^{2} A^{\prime \prime} /{ }^{4} A^{\prime \prime}{ }_{-}$, is also shown in Fig. 2 . The structure of MSX_ $C_{S_{-}}^{2} A^{\prime \prime} /{ }^{4} A^{\prime \prime}{ }_{-} 1$ is very similar to $D_{0_{-}} C_{S_{-}}$TS except that the $\overline{\mathrm{N}}^{1}-\mathrm{N}^{2}-\mathrm{N}^{3}$ angle is about $10^{\circ}$ smaller in MSX_ $C_{S_{-}}{ }^{2} A^{\prime \prime} I^{4} A^{\prime \prime}{ }_{-} 1$, which suggests that the crossing occurs on the isomerization coordinate before the TS. The energy of MSX_ $C_{S_{-}}{ }^{2} A^{\prime \prime} /{ }^{4} A^{\prime \prime}{ }_{-} 1$ is about $26.7 \mathrm{kcal} / \mathrm{mol}$ (uncorrected for ZPE) above the cyclic $\mathrm{N}_{3}$ minimum and $6.2 \mathrm{kcal} /$ mol (uncorrected for ZPE) below $D_{0_{-}} C_{S_{-}} \mathrm{TS}$, which is energetically similar to MSX_C $C_{2 v_{-}}{ }^{2} A_{2} /{ }^{4} B_{1}{ }_{-} 1$ but located at a different region of the PES. CASPT2 calculations give similar results with about $1 \mathrm{kcal} / \mathrm{mol}$ difference.

Spin-orbit interaction calculations at this MSX with the state-averaged CASSCF method shows that the norm of the coupling matrix is $20.90 \mathrm{~cm}^{-1}$, which comes exclusively from the interaction between two nonbonding $\pi$ orbitals, one is in-plane and the other is out-of-plane. The gradient difference for the bending motion is by far the largest, 0.228 a.u., at the MRCISD level. Adopting the same Laudau-Zener model with the same two kinetic-energy assumptions, one has $P=0.999$ and $P=0.998$ for the probability of spinconserving path, respectively. Similar to the MSX_ $C_{2 v_{-}}{ }^{2} A_{2} /{ }^{4} B_{1} 1$, the bending mode is mainly the driving force to bring the cyclic $\mathrm{N}_{3}$ to this crossing seam; therefore, we have the same lifetime as mentioned earlier.

Before we move to the next part, let us make a short summary concerning the spin-forbidden processes via the three MSXs we have presented, as they are responsible for the stability of the cyclic isomer. Clearly, dissociation through the seams of $\mathrm{MSX}_{-} C_{2 v_{-}}{ }^{2} A_{2} /{ }^{4} B_{1} 1$ or MSX_ $C_{S_{-}}{ }^{2} A^{\prime \prime} /{ }^{4} A^{\prime \prime}{ }_{-}$is not as effective as MSX_ $C_{2 v}{ }^{2} B_{1} /{ }^{4} A_{2}$ because of the relatively weak spinorbit coupling. The shortest lifetime in our estimation is about 5 ps which is short but detectable experimentally. However, one thing we should notice is that all these MSXs are located about $28 \mathrm{kcal} / \mathrm{mol}$ above the cyclic isomer. In other words, the molecule would not be able to enter into these strong interaction regions if the initial internal energy is substantially lower than $28 \mathrm{kcal} / \mathrm{mol}$, and as a consequence, the cyclic isomer will be long lived.

Now, let us look at the situation after the isomerization TS. In this region, the only quartet surface nearby is the lowest ${ }^{4} B_{1}$ state with a minimum located at $Q_{1_{-}}{ }^{4} B_{1}$, which results from a $C$-type Renner-Teller splitting. ${ }^{35}$ MSX optimization near this geometry located a $C_{2 v}$ structure, MSX_ $C_{2 v_{-}}{ }^{2} A_{2} /{ }^{4} B_{1} 2$, shown in Fig. 2. Geometrically, the structure of MSX_C $C_{2 v_{-}}{ }^{2} A_{2} / I^{4} B_{1} 2$ is very similar to that of $Q_{1_{-}}{ }^{4} B_{1}$, with less than $0.009 \AA$ and $7^{\circ}$ difference in 
$\mathrm{N}^{2}-\mathrm{N}^{1(3)}$ bond length and $\mathrm{N}^{1}-\mathrm{N}^{2}-\mathrm{N}^{3}$ bond angle, respectively, indicating the PESs cross near the quartet well's minimum. Energetically, MSX_C $C_{2 v}{ }^{2} A_{2} /{ }^{4} B_{1} 2$ is $47.4 \mathrm{kcal} / \mathrm{mol}$ above the linear $\mathrm{N}_{3}$ ground state and about $1.2 \mathrm{kcal} / \mathrm{mol}$ above the quartet minimum, $Q_{1}{ }^{4} B_{1}$ at the MRCISD level. Similar pictures are obtained by CASSCF and CASPT2, but slightly higher in energy.

When the earlier described strategy for the spin-orbit coupling evaluation was performed, one obtains $26.84 \mathrm{~cm}^{-1}$ for the norm of coupling matrix, which has the same type of interaction as that of MSX_ $C_{S_{-}}{ }^{2} A^{\prime \prime} /^{4} A^{\prime \prime}{ }_{-}$1. MRCISD gradient calculations indicate that the bending motion is the major contribution to the coupling with a magnitude of 0.175 a.u. at MSX_ $C_{2 v_{-}}{ }^{2} A_{2} /{ }^{4} B_{1}{ }_{2}$. Similarly, applying the same LaudauZener model with the same kinetic-energy assumption, one obtain probabilities of the spin-conserving path are $P$ $=0.998$ and $P=0.995$, respectively. Compared to previous MSXs, this channel may have larger contribution to the spinforbidden process, but small kinetic energy is still a prerequisite. Furthermore, it could be important for the quartet to doublet transition $\left(Q_{1_{-}}{ }^{4} B_{1} \rightarrow\right.$ linear- $\left.\mathrm{N}_{3}\right)$, because the crossing occurs many times in the quartet well, where the molecule may spend time and the cumulative transition probability could be appreciable.

\section{ISC in the vicinity of the linear dissociation TS}

Based on the discussion in previous sections, one knows that the quartet of the PES in this region is repulsive, and the energy of quartet state dissociation TS is lower than that of the doublet dissociation TS. Therefore, one could expect that the crossing occurs somewhere in between these two critical points. More specifically, the energy of quartet state will go up quickly with increasing $\mathrm{N}^{1}-\mathrm{N}^{2}-\mathrm{N}^{3}$ bond angle, and, finally, become degenerate with the doublet state. Actually, geometry optimization has located two minima on the crossing hypersurfaces, MSX_C $C_{S_{-}}{ }^{2} A^{\prime \prime} I^{4} A^{\prime \prime}{ }_{-} 2$ and MSX_ $C_{s_{-}}{ }^{2} A^{\prime} /{ }^{4} A^{\prime \prime}$ shown in Fig. 2, at all levels of theory used in the current study. Geometrically, the structures of these two MSXs are very close to that of the linear dissociation TS; the $\mathrm{N}^{1}-\mathrm{N}^{2}-\mathrm{N}^{3}$ angles in both MSXs are almost $180^{\circ}$ and the longer $\mathrm{N}-\mathrm{N}$ bonds are just about $0.3 \AA$ shorter than that of the dissociation TS. In addition, the energies of these two MSXs are very close to each other, a $0.4 \mathrm{kcal} / \mathrm{mol}$ difference at the MRCISD level. All the information suggests that PES crossings occur on the linear $\mathrm{N}_{3}$ dissociation coordinate slightly before the linear dissociation TS. The reason why there are two MSXs with similar energy is simply because the doubly degenerate $\Pi$ state will split along bending coordinate with corresponding symmetries. Energetically, MSX_ $C_{S_{-}}^{2} A^{\prime \prime} /{ }^{4} A^{\prime \prime}{ }_{-} 2$ is $52.5 \mathrm{kcal} / \mathrm{mol}$ relative to the groundstate linear $\mathrm{N}_{3}$, and just $0.02 \mathrm{kcal} / \mathrm{mol}$ higher in energy than that of MSX_ $C_{S_{-}}{ }^{2} A^{\prime} /^{4} A^{\prime \prime}$.

Spin-orbit calculations at state-averaged CASSCF level give the coupling magnitude of 57.6 and $57.4 \mathrm{~cm}^{-1}$ for the MSX_ $C_{S_{-}}{ }^{2} A^{\prime \prime} /^{4} A^{\prime \prime}{ }^{2}$ and MSX_ $C_{S_{-}}{ }^{2} A^{\prime} /^{4} A^{\prime \prime}$, respectively. For the MSX_ $C_{S_{-}}{ }^{2} A^{\prime \prime} I^{4} A^{\prime \prime}{ }_{-}$, MRCISD gradient difference is by far largest for motion along the direction of the $\mathrm{N}^{2}-\mathrm{N}^{3}$ distance with a value of 0.176 a.u., where $\mathrm{N}^{3}$ is the dissociating $\mathrm{N}$ atom. Also, assuming 1000 and $100 \mathrm{~cm}^{-1}$ kinetic energy along this coordinate, one obtains the Laudau-Zener probability of the spin-conserving path, $P=0.999$ and $P$ $=0.995$, respectively. For the MSX_ $C_{S_{-}}{ }^{2} A^{\prime} /^{4} A^{\prime \prime}$, the largest gradient difference of 0.176 a.u. is also found to be along the direction the $\mathrm{N}^{2}-\mathrm{N}^{3}$. Similar procedures give the probability of the spin-conserving path, $P=0.999$ and $P=0.995$, respectively.

\section{SUMMARY AND CONCLUSIONS}

In the present work, high-level $a b$ initio calculations have been carried out to study the ground and lowest quartet state of $\mathrm{N}_{3}$ radical both in the linear and cyclic regions, and the results can be summarized as follows:

(1) The structure and energetics of electronically ground and excited linear $\mathrm{N}_{3}$ radical have been determined at the CASSCF, CASPT2, and MRCISD(Q) levels of theory. Comparing with available experimental data and previous theoretical results, the current results at the MRCISD(Q) level of theory are closer to the experimental observation. The calculated vertical and adiabatic energies for $\widetilde{A}, \widetilde{B}$, and $\widetilde{a}$ states are very close to each other and indicate that a predissociation could easily occur in the photodissociation.

(2) The ground-state cyclic- $\mathrm{N}_{3}$ isomer has been identified and it is located $30.3 \mathrm{kcal} / \mathrm{mol}$ above the linear isomer at MRCISD(Q) level of theory. The isomerization transition state connecting these two isomers was determined to be at 63.4 and $32.1 \mathrm{kcal} / \mathrm{mol}$ above the linear and cyclic isomers, respectively. The local potential-energy surface around cyclic $\mathrm{N}_{3}$ was carefully investigated. A conical intersection between ${ }^{2} B_{1}$ and ${ }^{2} A_{2}$ states was located $13.2 \mathrm{kcal} / \mathrm{mol}$ above the cyclic- $\mathrm{N}_{3}$ minimum, and a $C_{2 v}$ transition state with $A_{2}$ symmetry was found to connect two equivalent cyclic- $\mathrm{N}_{3}$ minima but with different $C_{2}$ axis, i.e., pseudorotation.

(3) Adiabatic dissociation processes on the double and quartet states have been examined. A small dissociation barrier, $2.0 \mathrm{kcal} / \mathrm{mol}$, was found on the quartet state at the MRCISD(Q) level of theory. On the ground double surface, two dissociation barriers with respect to the cyclic and linear isomers were determined to be 33.1 and $59.6 \mathrm{kcal} / \mathrm{mol}$ also at the MRCISD $(\mathrm{Q})$ level of theory, respectively. However, the reverse barrier to form cyclic- $\mathrm{N}_{3}$ was found to be about 3.1 $\mathrm{kcal} / \mathrm{mol}$ higher than that of linear $\mathrm{N}_{3}$ formation, which implies that cyclic- $\mathrm{N}_{3}$ may not be able to be synthesized through the reaction of $\left.\mathrm{N}^{2} D\right)$ with $\mathrm{N}_{2}$ molecule.

(4) The nonadiabatic unimolecular dissociation processes have been carefully investigated. Six MSXs (minimum on the seam-of-crossing), which lead to the predissociation through the quartet state, were located on the dissociation and isomerization reaction coordinates. Spinorbit interaction and one-dimensional Laudau-Zener model calculations suggest that only near threshold, for which $v_{i}$ $\rightarrow 0$, will the probability of adiabatic, spin-forbidden behavior be appreciable.

(5) Perhaps the most important outcome of this work: the lowest MSX, MSX_ $C_{2 v_{-}}{ }^{2} B_{1} /{ }^{4} A_{2}$, lies $28.3 \mathrm{kcal} / \mathrm{mol}$ above the cyclic- $\mathrm{N}_{3}$ minimum, and combining the barriers for dissociation and isomerization, one could expect that cyclic- $\mathrm{N}_{3}$ is quite stable and long-lived under collision-free 
conditions, even if formed with a substantial internal energy. This provides strong theoretical support for the eventual isolation, detection, and characterization of this interesting molecule.

\section{ACKNOWLEDGMENTS}

This work was supported in part by grants from the Air Force Office of Scientific Research (Grant No. FA9550-041-0080) at Emory University and (Grant No. FA9550-04-10057) at the University of California, Santa Barbara. Computer resources were provided in part by the Cherry Emerson Center for Scientific Computation at Emory University as well as by a grant from AFOSR under the DoD High Performance Computing Program.

${ }^{1}$ J. P. Zheng, J. Waluk, J. Spanget-Larsen, D. M. Blake, and J. G. Radziszewski, Chem. Phys. Lett. 328, 227 (2000).

${ }^{2}$ T. J. Lee and J. M. L. Martin, Chem. Phys. Lett. 357, 319 (2002).

${ }^{3}$ J. P. Calfa, K. G. Phelan, and F. T. Bonner, Inorg. Chem. 21, 521 (1982); R. J. Gowland, K. R. Howes, and G. Stedman, J. Chem. Soc. Dalton Trans. 5, 797 (1992).

${ }^{4}$ A. Vij, J. G. Pavlovich, W. W. Wilson, V. Vij, and K. O. Christe, Angew. Chem., Int. Ed. 41, 3051 (2002).

${ }^{5}$ K. M. Dunn and K. Morokuma, J. Chem. Phys. 102, 4904 (1995); W. J. Lauderdale, J. F. Stanton, and R. J. Bartlett, J. Phys. Chem. 96, 1173 (1992); T. J. Lee and C. E. Dateo, Chem. Phys. Lett. 345, 295 (2001); T. J. Lee and J. E. Rice, J. Chem. Phys. 94, 1215 (1991); D. R. Yarkony, J. Am. Chem. Soc. 114, 5406 (1992); H. Ostmark, O. Launila, S. Wallin, and R. Tryman, J. Raman Spectrosc. 32, 195 (2001); M. N. Glukhovtsev, H. J. Jiao, and P. V. Schleyer, Inorg. Chem. 35, 7124 (1996); A. A. Korkin, A. Balkova, R. J. Bartlett, R. J. Boyd, and P. V. Schleyer, J. Phys. Chem. 100, 5702 (1996); M. Bittererova, T. Brinck, and H. Ostmark, J. Phys. Chem. A 104, 11999 (2000).

${ }^{6}$ M. Bittererova, H. Ostmark, and T. Brinck, J. Chem. Phys. 116, 9740 (2002).

${ }^{7}$ R. Klein and S. Biskupic, Chem. Pap.-Chem. Zvesti 47, 143 (1993).

${ }^{8}$ J. Wasilewski, J. Chem. Phys. 105, 10969 (1996).

${ }^{9}$ M. Bittererova, H. Ostmark, and T. Brinck, Chem. Phys. Lett. 347, 220 (2001); M. Bittererova, T. Brinck, and H. Ostmark, ibid. 340, 597 (2001); M. Tobita and R. J. Bartlett, J. Phys. Chem. A 105, 4107 (2001); A. Larson, M. Larsson, and H. Ostmark, J. Chem. Soc., Faraday Trans. 93, 2963 (1997); M. T. Nguyen and T. K. Ha, Chem. Ber. 129, 1157 (1996).
${ }^{10}$ M. L. Leininger, T. J. Van Huis, and H. F. Schaefer, J. Phys. Chem. A 101, 4460 (1997).

${ }^{11}$ F. Cacace, G. de Petris, and A. Troiani, Science 295, 480 (2002).

${ }^{12}$ L. E. Fried, M. R. Manaa, P. F. Pagoria, and R. L. Simpson, Annu. Rev. Mater. Sci. 31, 291 (2001).

${ }^{13}$ N. Hansen and A. M. Wodtke, J. Phys. Chem. A 107, 10608 (2003).

${ }^{14}$ A. Eppink and D. H. Parker, Rev. Sci. Instrum. 68, 3477 (1997).

${ }^{15}$ B. A. Thrush, Proc. R. Soc. London, Ser. A 235, 143 (1956).

${ }^{16}$ A. E. Douglas and W. J. Jones, Can. J. Phys. 43, 2216 (1965).

${ }^{17}$ R. A. Beaman, T. Nelson, D. S. Richards, and D. W. Setser, J. Phys. Chem. 91, 6090 (1987).

${ }^{18}$ C. R. Brazier, P. F. Bernath, J. B. Burkholder, and C. J. Howard, J. Chem. Phys. 89, 1762 (1988).

${ }^{19}$ R. Pahnke, S. H. Ashworth, and J. M. Brown, Chem. Phys. Lett. 147, 179 (1988).

${ }^{20}$ R. J. Tian, J. C. Facelli, and J. Michl, J. Phys. Chem. 92, 4073 (1988).

${ }^{21}$ R. E. Continetti, D. R. Cyr, R. B. Metz, and D. M. Neumark, Chem. Phys. Lett. 182, 406 (1991).

${ }^{22}$ R. E. Continetti, D. R. Cyr, D. L. Osborn, D. J. Leahy, and D. M. Neumark, J. Chem. Phys. 99, 2616 (1993).

${ }^{23}$ M. J. Pellerite, R. L. Jackson, and J. I. Brauman, J. Phys. Chem. 85, 1624 (1981).

${ }^{24}$ C. Petrongolo, J. Mol. Struct. 175, 215 (1988).

${ }^{25}$ J. M. L. Martin, J. P. Francois, and R. Gijbels, J. Chem. Phys. 90, 6469 (1989).

${ }^{26}$ J. M. L. Martin, J. P. Francois, and R. Gijbels, J. Chem. Phys. 93, 4485 (1990).

${ }^{27}$ R. A. Kendall, T. H. Dunning, and R. J. Harrison, J. Chem. Phys. 96, 6796 (1992).

${ }^{28}$ P. J. Knowles and H.-J. Werner, Chem. Phys. Lett. 115, 259 (1985).

${ }^{29}$ P. Celani and H.-J. Werner, J. Chem. Phys. 112, 5546 (2000).

${ }^{30}$ P. J. Knowles and H.-J. Werner, Chem. Phys. Lett. 145, 514 (1988).

${ }^{31}$ E. R. Davidson, J. Comput. Phys. 17, 87 (1975); S. R. Langhoff and E. R. Davidson, Int. J. Quantum Chem. 8, 61 (1974).

${ }^{32}$ D. G. Fedorov and M. S. Gordon, J. Chem. Phys. 112, 5611 (2000).

${ }^{33}$ M. W. Schmidt, K. K. Baldridge, and J. A. Boatz, J. Comput. Chem. 14, 1347 (1993).

${ }^{34}$ N. Koga and K. Morokuma, Chem. Phys. Lett. 119, 371 (1985).

${ }^{35}$ G. Herzberg, Electronic Spectra of Polyatomic Molecules (Van Nostrand, New York, 1966).

${ }^{36}$ R. Prasad, J. Chem. Phys. 119, 9549 (2003).

${ }^{37}$ NIST Atomic Spectra Database.

${ }^{38}$ M. Desouter-Lecomte and J. C. Lorquet, J. Chem. Phys. 66, 4006 (1977).

${ }^{39} \mathrm{MOLPRO}$ is a package of $a b$ initio programs written by H.-J. Werner, P. J. Knowles, M. Schütz, et al. . 